\title{
GESTIÓN DE LA PRIVACIDAD DE LOS PERFILES DE FACEBOOK DEADOLESCENTES
}

\section{PRIVACY MANAGEMENT IN FACEBOOK PROFILES OF ADOLESCENTS}

\author{
Dr. Andrés Chamarro Lusar ${ }^{1}$ \\ andres.chamarro@uab.cat \\ Enric Bertran Martí1 \\ enric.bertran.marti@gmail.com \\ Dra. Úrsula Oberst ${ }^{2}$ \\ ursulao@blanquerna.edu \\ Alexandra Torres Rodríguez ${ }^{2}$ \\ alexandratr@blanquerna.edu
}

${ }^{1}$ Universidad Autónoma de Barcelona. Facultad Psicología Básica.

Departamento de Psicología Básica, Evolutiva y de la Educación.

Edificio B. Campus de Bellaterra, 08193, Cerdanyola del Vallés, Barcelona (España)

${ }^{2}$ Universidad Ramon Llull. Facultad de Psicología, Ciencias de la Educación y del Deporte Blanquerna. Departamento de Psicología. c/ Cister 34, 08002, Barcelona (España)

Este estudio analiza el estilo de privacidad en Facebook. Participaron 373 alumnos de entre 13 y 17 años que respondieron a un cuestionario sobre la privacidad en Facebook. Emergieron tres patrones de privacidad, correspondientes con un estilo protector, en el que los usuarios solo reciben mensajes de amigos y de amigos de amigos; restringido, en el que los adolescentes están abiertos a conocer nuevos amigos; y expuesto, en el que destaca la exposición de la información y contenidos abiertos a todo el público. A medida que los adolescentes dominan el uso de Facebook, también adquieren mayor consciencia de privacidad.

Palabras clave: Redes Sociales Online, Facebook, privacidad, adolescència.

This study analyzes privacy styles on Facebook. They involved 373 students ages 13 to 17 who answered a questionnaire about privacy in Facebook. The emerged three patterns of privacy corresponding with a protective style, in which users receive messages from friends, and friends of friends; restrictive style, in which teenagers are open to meet new friends; and exposed style, in which users had personal information and content with access for everyone. As teenagers become the dominant use of Facebook, also acquire greater conscience of privacy

Keywords: Online Social Networks, Facebook, privacy, adolescence. 


\section{Introducción.}

La navegación por Internet, el uso de redes sociales online (RSO), los videojuegos y el teléfono móvil han supuesto un cambio radical en las formas de relacionarse de la mayoría de las personas. Para los más jóvenes, que acceden a ellas desde niños, probablemente estén marcando un nuevo estilo de vida (Muñoz et al., 2014). Los estudios sobre el efecto del uso de Internet, videojuegos y teléfono móvil sobre los adolescentes son frecuentes, y se están extendiendo al uso de redes sociales on-line (RSO).

Las RSO, como MySpace, Facebook, Twitter, Linkedin y Whatsapp, son comunidades virtuales en las que las personas pueden crear su propio perfil con todo tipo de información personal e interactuar con los perfiles de sus amigos en la vida real, así como conocer a nuevas personas con las que compartan algún interés (Boyd \& Ellison, 2007). Una de las redes sociales que ha causado un mayor impacto social ha sido Facebook, que es considerada la RSO por excelencia (Davenport, Bergman, Bergman \& Fearrington, 2014; Observatorio Nacional de las Telecomunicaciones y de la Sociedad de la Información, 2013), con catorce millones de usuarios estimados en España (Interactive Advertising Bureau, 2015). Ésta fue fundada en 2004 como una herramienta de comunicación para los estudiantes de Harvard y desde 2006, cuando fue abierta al público en general, se ha convertido en la red social líder y más popular en todo el mundo. En el 2012 tenía mil millones de usuarios activos mensualmente ( $\mathrm{B}^{3}$ achnio, Przepiórka \& Rudnicka, 2013), con una alta prevalencia entre adolescentes y estudiantes universitarios (Ellison, Steinfield \& Lampe, 2007). Facebook permite a sus usuarios no sólo comunicarse, sino también compartir fotos, jugar, y hasta revelar información personal, como el correo electrónico, la fecha de nacimiento, creencias religiosas o políticas, la ocupación laboral y el estado civil. Es, por tanto, un espacio con multiplicidad de aplicaciones, que inicialmente estaban centradas en la comunicación y el entretenimiento, pero que recientemente se ha convertido en un espacio interesante para la política o la economía, puesto que es fuente de información para los negocios y el marketing (Blachnio et al., 2013). De hecho, uno de cada cuatro usuarios siguen a marcas comerciales en su RSO (Interactive Advertising Bureau, 2015).

Desde el punto de vista del uso, en Facebook la mayoría de amigos se conocen y luego son invitados a convertirse en miembros del grupo de amigos en línea (Ross et al., 2009), que puede fácilmente alcanzar una magnitud de entre 200 y 250 «amigos» (Almansa, Fonseca \& Castillo, 2013; Kalpidou, Costin \& Morris, 2011). En todo caso, no son descartables magnitudes superiores; por ejemplo, Christofides, Muise y Desmarais (2009) encontraron que los estudiantes universitarios tenían una media de 297 «amigos» en Facebook. Dado que Facebook ha generado una nueva forma de relación social, la privacidad de la información que sus usuarios depositan en ella es un tema que merece atención (Casado, Oberst \& Carbonell, 2015).

Existe un cuerpo creciente de estudios que evalúan la repercusión que tiene este tipo de comunicación en la vida de los adolescentes. En general, las RSO facilitan la relación social entre iguales, que es muy importante en la adolescencia, puesto que permite procesos de aprendizaje social, maduración y desarrollo (Boyd, 2014). Facebook precipita esta 
iniciación en lo social, haciendo que se mantengan relaciones accesibles las veinticuatro horas. A ello hay que añadir que cada persona puede hacer uso de Facebook según sus inquietudes y necesidades, eligiendo las relaciones que establece y los grupos con los que se relaciona (Cheung \& Lee, 2010; Kwon \& Wen, 2010). Este elevado impacto social ha generado un incremento del interés científico por las RSO, con estudios sobre las motivaciones de unirse a Facebook (B³achnio et al., 2013; Caers et al., 2013), hábitos de uso (García, López de Ayala \& Catalina, 2013; Linne, 2014) los efectos en la identidad (Boyd, 2014; Renau, Oberst \& Carbonell, 2013), las diferencias de género en el uso (Renau, Carbonell \& Oberst, 2012), las características de los usuarios (Caers et al., 2013; Shen, Brdiczka \& Ruan, 2013), y la privacidad (Boyd, 2014; Caers et al., 2013) Una reciente revisión de literatura concluye que un $24 \%$ de los artículos analizan a los usuarios, un $19 \%$ las motivaciones para usar Facebook, un $12 \%$ la identidad de los usuarios, un $27 \%$ el rol de Facebook en interacciones sociales y un $18 \%$ la privacidad y la revelación de la información (Wilson, Gosling \& Graham, 2012). Respecto a la intensidad de uso, Christofides et al. (2009) encontraron que los jóvenes dedican una media de 38 minutos diarios a publicar en Facebook. Raacke y Bonds (2008) mostraron que los estudiantes que tenían cuentas en varias RSO, incluyendo Facebook, dedicaron un promedio de 3 horas al día a publicar mensajes.

\subsection{Privacidad en las redes sociales.}

El rápido crecimiento de las $\mathrm{RSO}$ ha coincidido con una creciente preocupación sobre la privacidad personal, desencadenada por la alarma social acerca de los peligros a los que un adolescente está expuesto a través de Internet y las RSO (Lewis, Kaufman \& Christakis, 2008). Los riesgos más habituales que se han desarrollado con la expansión del uso de Internet entre menores y adolescentes son: a) Adicción a las nuevas tecnologías; b) Ciberacoso sexual; c) Acceso a contenido poco fiable; d) Ciberbullying; e) Malinterpretación de la información social; f) Compartir información de otras personas; g) Publicar contenidos inadecuados; h) Pérdida de la anonimidad, etc. (Acquisti \& Gross, 2006; Merritt, 2008). Estos riesgos son especialmente importantes entre los adolescentes si consideramos que éstos se caracterizan por priorizar la gratificación inmediata y el vivir el presente, creerse invulnerables al peligro, sentir curiosidad por nuevas vivencias estimulantes y arriesgadas, tener dificultad en el control de los impulsos y ser influenciables por los medios de comunicación y la publicidad, lo que los hace más susceptibles a las conductas de riesgo (Muñoz et al., 2014). Christofides et al. (2009) consideran que un riesgo esperable entre los jóvenes usuarios de Facebook es sentirse incómodo o lamentar la divulgación de información privada y experimentar conflictos con los padres. En este contexto, los adolescentes constituyen un grupo de alto riesgo al ser los más familiarizados con Internet y a las redes sociales (Observatorio Nacional de las Telecomunicaciones y de la SI, 2013). Es por ello que la privacidad juega un papel clave en la protección de los adolescentes respecto a estos riesgos.

La gestión de la privacidad hace referencia a las opciones de configuración que hacen los usuarios en Facebook y otras RSO, para controlar la información que quieren mostrar en su perfil y su visibilidad. La difusión 
considerable de información que dan a conocer los usuarios y su descontextualización puede generar consecuencias negativas, como contactos indeseados, divulgación indeseada de información y malentendidos, lo que supone una amenaza a la privacidad (Casado et al., 2015; Christofides, Muise \& Desmarais, 2012). La política de uso de datos en ocasiones genera desconocimiento y ha generado un debate sobre la seguridad de la información en las RSO. Según el Instituto Nacional de Tecnologías de la Información, INTECO (2012), los usuarios en las RSO solamente permiten acceso a su perfil privado a sus contactos, gestionando así la privacidad de su perfil, aunque en ocasiones dicha gestión se torna complicada para un $42,5 \%$ de usuarios. Sin embargo, no queda claro si el uso de la privacidad es el más adecuado. Autores como Ellison et al., (2007) consideran que los participantes de las RSO están relativamente abiertos a publicar información personal y tienen una cierta falta de conciencia sobre la importancia de la privacidad. Una baja conciencia respecto a la privacidad y la protección de los datos de carácter personal en Internet desencadena múltiples riesgos. Christofides et al. (2009) consideran que los usuarios de RSO pueden tener una conducta de protección de la privacidad diferente a la esperable, presentando mucha información personal sin calcular el impacto que puede tener y a quién deberían restringir o autorizar su consulta. Además, para los adolescentes compartir información puede ser utilizado como una forma de generar confianza entre los miembros de su red, de los que se espera un comportamiento equivalente.

Son pocos los estudios empíricos acerca de las prácticas de privacidad de los usuarios de las RSO y aún es menos común que se recojan datos de menores y adolescentes en edad escolar. Los escasos estudios existentes, muestran que los jóvenes universitarios son propensos a tener perfil privado si los amigos/ compañeros tienen sus perfiles privados, si se es más activo en Facebook y si se es mujer (Junco, 2012; Lewis, Kaufman \& Christakis, 2008; Nadkarni \& Hofmann, 2012). En todo caso, Kuss y Griffiths (2011) consideran que las evidencias respecto al uso diferencial según el sexo son contradictorias. Christofides, Muise y Desmarais (2011) encontraron que los estudiantes eran conscientes de la importancia de su privacidad en Facebook y utilizaban la configuración de privacidad. Pese a ello, la necesidad de obtener popularidad les hacía mostrar más información en Facebook de la que mostrarían en su vida privada, lo que los autores consideran una práctica de riesgo. Los autores concluyen que esto está influenciado por las normas implícitas acerca de lo que se considera adecuado: ser proactivo a la hora de compartir información y responder a lo que otros comparten. Waters y Ackerman (2011) encontraron que revelar información privada en la RSO respondía a interés por almacenar información o entretenimiento, compartir información, mantenerse al día con las tendencias y, por último, mostrar popularidad.

En los adolescentes se espera un inicio temprano en el uso de RSO, entre los 12 y 13 años, por motivos de curiosidad, comunicación y socialización, utilizando estrategias de ensayo-error. Este inicio temprano se da por la baja ansiedad que implica la relación on-line y los beneficios que aporta: a) Autorrevelación al realizar un perfil, decidiendo qué elementos de la identidad se hacen destacables y cómo se comunican; y b) Oportunidad de unirse a grupos y mejorar 
los sentimientos de pertenencia (Shapiro \& Margolin, 2014). Merritt (2008), ha propuesto que la baja privacidad es característica de esta época, hasta que no se sufren las consecuencias de ésta. A los 14 y 15 años, se incrementa la necesidad de comunicación entre iguales, con la aparición de la necesidad de formar parte permanente de las redes sociales, y se produce un desplazamiento de su uso a los smartphones. A los 16 años y más, aparece la conciencia sobre la privacidad y los riesgos en las redes sociales, fruto de la propia experiencia y la experiencia vicaria. Christofides et al. (2009) exploraron el uso que los adolescentes hacen de las opciones para proteger la privacidad en su perfil y hallaron que muestran más información personal y utilizan menos las opciones de la configuración de la privacidad de Facebook que los adultos. En definitiva, parece que los adolescentes están en una situación de potencial vulnerabilidad en el uso de las RSO. Limitar esta vulnerabilidad puede ser una tarea relativamente urgente si consideramos su crecimiento actual y el aumento previsible con la expansión de los smartphones con acceso a Internet.

En base a lo presentado hasta ahora, y para contribuir a la protección de la privacidad de los menores, en este estudio nos proponemos estudiar las configuraciones de privacidad de los adolescentes. Concretamente, nos proponemos un triple objetivo: primero, caracterizar el estilo de privacidad en Facebook de los adolescentes españoles, dada la escasez de estudios empíricos al respecto; segundo, determinar la prevalencia del uso de riesgo para la privacidad que se da entre los adolescentes; y tercero, establecer relación entre los estilos de privacidad y variables sociodemográficas y de uso.

\section{Material y métodos.}

\subsection{Participantes y procedimiento.}

Los participantes fueron alumnos de seis institutos de enseñanza secundaria de Cataluña que asistieron a talleres educativos sobre uso seguro de Facebook. En total se analizaron los datos de 373 participantes con perfil personal en Facebook. El 51,5\% de la muestra eran mujeres. La franja de edad estaba comprendida entre los 12 y los 16 años $(\mathrm{M}=$ $13,96, \mathrm{SD}=1,18)$. Los participantes en el momento de responder el cuestionario, estaban estudiando Primero de ESO (Educación Secundaria Obligatoria) (15,5\%), Segundo de ESO (26,0\%), Tercero de ESO $(30,6 \%)$ y Cuarto de ESO $(27,9 \%)$. Los padres o tutores de los alumnos y la dirección del centro autorizaron el estudio mediante consentimiento informado.

\subsection{Instrumentos y procedimiento.}

La recogida de datos fue llevada a cabo entre enero y abril de 2014. Antes de comenzar el taller educativo, los alumnos podían contestar, de forma anónima y totalmente voluntaria (sólo tenían que indicar su sexo y su edad), un cuestionario construido ad hoc sobre el uso que hacían de la privacidad de su perfil en Facebook. El cuestionario constaba de 18 ítems con 2 a 6 opciones de respuesta (según el caso) que interrogaban sobre la antigüedad del perfil en Facebook (menos de 1 año; entre 1 y 2 años; más de 2 años), tiempo de uso semanal (menos de media hora; entre media hora y 2 horas; entre 2 y 4 horas; más de 4 horas), personas que tienen acceso a mensajería, publicaciones y perfil (p.ej., ¿Quién puede enviarte mensajes de Facebook?; con respuestas: público, 
amigos de amigos, amigos), opciones de privacidad (p.ej., revisión de la biografía/perfil; con respuestas: activada, desactivada), acceso a información personal (p.ej., ¿Quién puede ver dónde estudias?; con respuestas: Público, Amigos, Sólo yo, Personalizado, No lo tengo puesto) e inclusión parental en Facebook y restricción de información a estos.

\subsection{Análisis de datos.}

Se calculó un indicador de privacidad sumando las respuestas de los participantes a los ítems de estado, visibilidad, privacidad de su perfil y de sus diferentes opciones.

Se llevó a cabo un análisis de clúster no jerárquico (K-means) para determinar la existencia de grupos homogéneos de usuarios de Facebook con respecto a la privacidad. Adicionalmente, se calcularon sucesivos análisis de varianza unifactoriales con contrastes a posteriori, aplicando el test de Bonferroni para comparaciones de medias por pares (de dos en dos), para las diferencias en los patrones de privacidad según edad, sexo, antigüedad de uso y horas de uso semanal.

\section{Análisis y resultados.}

Como se puede observar en la Tabla 1, la mayoría de los participantes hacía más de 2 años que disponían de un perfil, seguidos por los que hacía entre 1 y 2 años y los que hacía menos de un año. Los estudiantes a partir de $2^{\circ}$ de ESO muestran una antigüedad superior a los dos años $(\div 2=44,28 ; p<, 001)$. No se observan diferencias de sexo $(\div 2=2,60$; $p>, 05)$. Respecto al uso semanal había $29,5 \%$ que le dedicaba menos de media hora, un $26,3 \%$ que dedicaba entre media y 2 horas, un $13,1 \%$ que dedicaba de 2 a 4 horas y un $14,5 \%$ que dedicaba más de 4 horas a la semana. No se aprecian diferencias significativas en el tiempo de uso semanal para los cursos $(\div 2=9,65 ; p>, 05)$, ni para el $\operatorname{sexo}(\div 2=1,85 ; p>, 05)$.

El análisis clúster mostró una solución óptima de 3 factores. Los estadísticos descriptivos de cada clúster se presentan en la Tabla 2.

El clúster 1, agrupa a participantes que muestran un perfil que hemos denominado «Protector», ya que destaca por ser el grupo con una protección superior de datos. Los usuarios en este tipo de perfil solo reciben mensajes de amigos y de amigos de amigos. De cara a los permisos de información y contenidos, sólo ellos pueden ver donde estudian y las fotos de sus propios álbumes y no tienen habilitada la opción de estado sentimental.

El clúster 2, agrupa a participantes que muestran un perfil que hemos considerado «Restringido». Este perfil se caracteriza por estar abierto a conocer nuevos amigos a través de Facebook. El perfil sólo puede recibir solicitudes de amistad de amigos de amigos y solo puede recibir mensajes privados de amigos. Las publicaciones de su perfil las pueden ver los amigos, también saber dónde estudian está restringido a amigos.

El clúster 3, denominado «Expuesto», destaca por la exposición de su información y contenidos abiertos a todo el público. Cualquier usuario puede enviarle mensajes, y en su página de perfil permite publicar a amigos y amigos de amigos. La información de dónde estudia es pública. También es el único perfil en el que tiene a algún usuario bloqueado.

Como se puede apreciar en la Tabla 2, el ANOVA reveló que no había diferencias significativas en los patrones de privacidad para los diferentes grupos de edad, curso y sexo. Sí que había diferencias significativas 


\begin{tabular}{|c|c|c|c|c|c|c|c|}
\hline \multirow[b]{2}{*}{$\begin{array}{c}\text { CURSO } \\
\text { DE } \\
\text { ESO }\end{array}$} & \multicolumn{3}{|c|}{ ANTIGÜEDAD DE USO } & \multicolumn{4}{|c|}{ USO SEMANAL } \\
\hline & $<1$ año & $\begin{array}{l}\text { Entre } 1 \text { y } 2 \\
\text { años }\end{array}$ & $>2$ años & $<1 / 2$ hora & $\begin{array}{l}\text { Entre } 1 / 2 \text { hora } \\
\text { y } 2 \text { horas }\end{array}$ & $\begin{array}{l}\text { Entre } 2 \text { y } 4 \\
\text { horas }\end{array}$ & $>4$ horas \\
\hline $1^{\circ}$ & $13(22,4 \%)$ & $14(24,1 \%)$ & $31(53,4 \%)$ & $15(31,3 \%)$ & $16(33,3 \%)$ & $8(16,7 \%)$ & $9(18,8 \%)$ \\
\hline $2^{\circ}$ & $5(5,2 \%)$ & $28(26,0 \%)$ & $63(65,6 \%)$ & $27(34,2 \%)$ & $25(31,6 \%)$ & $12(15,2 \%)$ & $15(19,0 \%)$ \\
\hline $3^{\circ}$ & $6(5,4 \%)$ & $22(19,6 \%)$ & $84(75,0 \%)$ & $39(42,4 \%)$ & $24(26,1 \%)$ & $19(20,7 \%)$ & $10(10,9 \%)$ \\
\hline $4^{\circ}$ & $3(2,9 \%)$ & $7(6,7 \%)$ & $94(90,4 \%)$ & $29(31,5 \%)$ & $33(35,9 \%)$ & $10(10,9 \%)$ & $20(21,7 \%)$ \\
\hline Total & $27(7,3 \%)$ & $71(19,2 \%)$ & $272(73,5 \%)$ & $110(35,3 \%)$ & $98(31,5 \%)$ & $49(15,8 \%)$ & $54(17,4 \%)$ \\
\hline
\end{tabular}

Tabla 1. Número (entre paréntesis, el porcentaje) de alumnos según antigüedad y tiempo de uso.

\begin{tabular}{|c|c|c|c|c|c|c|}
\hline \multicolumn{2}{|c|}{ VARIABLE } & $\begin{array}{c}\text { CLÚSTER 1 } \\
(N=99,26,26 \%)\end{array}$ & $\begin{array}{c}\text { CLÚSTER 2 } \\
(N=155,41,11 \%)\end{array}$ & $\begin{array}{c}\text { CLÚSTER 3 } \\
(N=119,31,56 \%)\end{array}$ & & \\
\hline $\begin{array}{l}\text { Perfil de } \\
\text { clúster }\end{array}$ & Rango & $M(D T)$ & $M(D T)$ & $M(D T)$ & $F$ & $p$ \\
\hline Edad & $12-16$ & $13,80(1,27)$ & $13,99(1,11)$ & $14,05(1,19)$ & 1,35 & 261 \\
\hline Curso & $1-4$ & $2,57(1,11)$ & $2,81(0,97)$ & $2,70(1,05)$ & 1,64 & , 196 \\
\hline Antigüedad & $1-3$ & $2,53(0,675)$ & $2,65(0,63)$ & $2,79(0,49)$ & 5,16 & ,006 \\
\hline $\begin{array}{l}\text { Uso } \\
\text { semanal }\end{array}$ & $1-4$ & $2,00(1,06)$ & $1,99(1,01)$ & $2,52(1,15)$ & 7,77 & ,001 \\
\hline \multicolumn{2}{|l|}{ Frecuencias } & $N(\%)$ & $N(\%)$ & $N(\%)$ & & \\
\hline \multirow[t]{2}{*}{ Sexo } & Mujer & $49(49,50 \%)$ & $80(51,60 \%)$ & $52(43,70 \%)$ & 87 & 42 \\
\hline & Hombre & $50(50,50 \%)$ & $75(48,40 \%)$ & $67(56,30 \%)$ & & \\
\hline
\end{tabular}

Tabla 2. Estadísticos descriptivos y comparaciones para los clústeres.

en los patrones de uso en función de la antigüedad de uso y las horas de uso semanal. Las comparaciones por pares indican que las diferencias se observan para la antigüedad de uso entre los alumnos que hace más de 2 años, que muestran el perfil más expuesto. Para el uso semanal las diferencias se observan entre los alumnos que dedican más de 4 horas a la semana, que también muestran el perfil más expuesto.

\section{Discusión.}

El presente estudio tenía por objetivo caracterizar el estilo de privacidad en Facebook de los adolescentes, determinar la prevalencia del uso de riesgo, y analizar la relación entre los estilos de privacidad y variables sociodemográficas y de uso. Respecto al primer objetivo, nuestros resultados muestran que los adolescentes adoptan tres estilos principales a la hora de establecer la privacidad de su perfil en Facebook. Estos tres estilos parecen corresponderse con diferentes estadios en el uso de Facebook. En el primer patrón los adolescentes parecen adoptar medidas de protección de la privacidad, en el segundo se va produciendo una abertura progresiva del perfil, y en el tercero el adolescente ya abre públicamente su perfil.

Respecto a la prevalencia, un 31,56\% de nuestra muestra de adolescentes muestra un patrón de alta exposición de sus contenidos a la vista de extraños. Esta prevalencia puede ser considerada muy elevada, pese a la ausencia de datos de prevalencia comparables, si consideramos la franja de edad de nuestros participantes. Los indicios más próximos los encontramos en la prevalencia de conducta de riesgo en el uso de Facebook en adolescentes franceses, que es del $12 \%$ en chicas y del $5 \%$ en chicos (Delfour, Moreau, Laconi, Goutaudier \& 
Chabrol, 2014). Si bien en nuestro estudio no se analiza directamente conducta de riesgo en el uso de Facebook, cabe esperar que un porcentaje indeterminado de los adolescentes que protegen poco los contenidos de su perfil muestren conductas de riesgo. En este sentido, Kuss y Griffiths (2011) han mostrado como el uso intensivo y la exposición de contenido personal, que puede ser fuente de conflictos, son característicos del uso de riesgo de las redes sociales. Futuros estudios deberían estudiar esta relación.

En todo caso, esta elevada prevalencia del estilo «Expuesto» puede ser debida, al menos en parte, a la generalización de las RSO, su cada vez más fácil acceso desde los smartphones y la familiaridad en el uso de las RSO. Christofides et al. $(2009,2011)$ ya han mostrado recientemente que los adolescentes utilizan menos opciones de privacidad que los adultos. Así pues, la conducta de riesgo en el uso de las RSO es un nuevo factor condicionante de la salud de la población, que se debe tener en cuenta sobre todo en la atención a los adolescentes.

Respecto a la relación entre los patrones de privacidad y las variables analizadas, en primer lugar cabe destacar el patrón de uso de RSO de nuestra muestra, que pone de relieve cómo cuando inician la ESO más de un $50 \%$ de los adolescentes ya tiene perfil de Facebook, y cómo su uso se generaliza en los años posteriores, independientemente del sexo. Este uso generalizado contrasta con el escaso tiempo dedicado, menos de dos horas a la semana. Estos resultados contrastan con el elevado uso informado por otros estudios con adolescentes, especialmente entre las chicas (Muñoz et al., 2014). Nuestros resultados también indican que los tres patrones de privacidad son progresivos, de mayor a menor privacidad, y no parecen estar relacionados con la edad, el curso y el sexo.
Por el contrario, parecen estar asociados a la antigüedad y la frecuencia de uso de Facebook. Los resultados son ciertamente sugerentes y ponen de relieve que un inicio temprano en las RSO es independiente del género y de la edad. Estos resultados contradicen la investigación previa respecto al uso diferencial de las redes sociales por parte de los chicos y chicas (Kuss \& Griffiths, 2011), y las propuestas de Merritt (2008) de que los adolescentes adquieren conciencia de la privacidad y sus riesgos con la edad. De hecho, nuestros resultados muestran una progresiva menor observancia de las pautas de privacidad, justo al contrario que las recomendaciones de las autoridades y que educadores intentan promover entre los adolescentes. Esta práctica es diferente a la observada entre adolescentes de otros estudios (Junco, 2012; Lewis, Kaufman \& Christakis, 2008). Parece que los adolescentes que muestran el perfil «expuesto» no tuvieran una gran conciencia de la importancia de su privacidad en Facebook y que un mayor grado de familiarización con este medio conlleva a una menor precaución respecto a quien puede ver el contenido. El hecho de que sea el único perfil que tiende a bloquear usuarios no deseados, para que no tengan acceso a su información, nos muestra patrones de conducta más reactivos que preventivos. En definitiva, parecería que a medida que ganan experiencia en el uso de Facebook se tiende hacia el descuido y la despreocupación. Posiblemente, esto tenga que ver con la generación estudiada, una generación «nativa digital» (Bennett, Maton \& Kervin, 2008; Prensky, 2001; Selwyn, 2009) que ha crecido con las RSO y que puede tener actitudes, motivaciones y prácticas de privacidad diferentes a las anteriores (Davenport et al. 2014). Esto puede ir también asociado a los cambios en la política de 
privacidad de Facebook, que a lo largo de su existencia, ha experimentado cambios. A raíz de quejas de la sociedad y de los medios de comunicación, Facebook, en 2010, modificó (y simplificó) las opciones de ajuste de la privacidad, mediante las cuales, el usuario puede elegir con quien quiere compartir su información: con amigos, amigos de amigos, con todo el mundo, o una opción «recomendada» que combina las tres anteriores. Así, podría ser que los adolescentes con el perfil «expuesto» simplemente se limiten a seguir las recomendaciones de privacidad de su RSO preferida. En todo caso, como Christofides et al. (2012) han destacado, los controles de privacidad en las RSO suelen ser difíciles de entender, incluso para los adultos.

Otra explicación alternativa sería que a medida que ganan experiencia en el uso de las RSO adquieren más dominio respecto a la calidad de la información que presentan, de tal manera que su exposición pública y sin restricciones no sea considerada comprometedora. Esto es consistente con la sugerencia de Christofides et al. (2009), que consideran que Facebook ha contribuido a la aparición de una nueva norma implícita acerca de lo que se considera adecuado en las RSO: ser popular. Ser popular implica ser proactivo a la hora de compartir información y responder a lo que otros comparten, haciendo que la divulgación de información sea el factor clave en la evaluación de una persona. Desde este punto de vista, tener una presencia en Facebook requiere que una persona sea activa y publique con frecuencia. De este modo, la popularidad y la divulgación se convierten en dos aspectos vinculados. Como resultado, limitar el acceso a la información personal reduce potencialmente la popularidad, y para los adolescentes ser popular y recibir apoyo de sus pares es una cosa muy valorada (Waters \& Ackerman, 2011). Ellison et al. (2007) también mostraron que el uso intensivo de Facebook es un medio para adquirir capital social, relaciones, en momentos de transición vital, como el cambio de centro de estudios. Shen, Brdiczka y Ruan (2013) muestran cómo Facebook se utiliza más para la expresión de contenidos emocionales, en comparación con otros medios de comunicación digital, como el correo electrónico.

Los resultados de este estudio deben ser considerados con cautela, especialmente si consideramos que los participantes son voluntarios, de una única zona geográfica (Cataluña) y sin datos relativos acerca de características relevantes como el origen, la clase social, la tipología de centro, etc. Futuros estudios deberían realizarse con muestras más amplias y mejor caracterizadas. Otra limitación tiene que ver con la ausencia de datos respecto a los contenidos de los perfiles de Facebook. Futuros estudios deberían analizar la calidad de la información personal que ofrecen los adolescentes en sus perfiles, que sí que podrían estar relacionados con el sexo de sus propietarios y con prácticas de riesgo. En este sentido, Davenport et al. (2014) han puesto de relieve que medidas de uso como el «tiempo pasado» en la plataforma son poco precisas y obvian que el uso de las RSO puede ser «activo», por ejemplo, realizando actualizaciones de estado y comentarios o «pasivo», en que la participación es únicamente como un consumidor de contenido (por ejemplo, la lectura de los mensajes, ver fotografías, etc.). Futuros estudios deberían explorar la relación entre los patrones de privacidad, las diferencias en el tipo de uso y el género, puesto que podría ser que el uso preferente de las RSO por parte de las chicas se haya generalizado, tal como sugieren nuestros 
resultados. Finalmente, otra limitación importante es la relativa al diseño transversal de este estudio. Recoger información longitudinalmente sería una forma directa de ver la evolución del uso de Facebook y las redes sociales.

\section{Conclusiones.}

Este estudio muestra, de forma innovadora, cuales son los estilos de privacidad en el uso de Facebook de una muestra de adolescentes. Los resultados ponen de relieve que las prácticas de privacidad no dependen de factores como la edad y el sexo, sino que parecen ir asociados a aspectos relativos a la experiencia. Pese a que la exposición pública de contenidos personales en el perfil de Facebook sea una práctica de riesgo potencial respecto a la privacidad, consideramos que la importante experiencia acumulada por los adolescentes y su utilidad para estar en contacto con los amigos, mejorar la influencia social, disminuir la sensación de soledad y proyectar una imagen positiva de uno mismo, explican en buena medida la elevada prevalencia del estilo «expuesto» en su uso. En todo caso, creemos que son necesarias unas instrucciones claras acerca de las opciones de privacidad, y una formación específica sobre su uso e implicaciones que permita a los adolescentes tener un mejor control sobre la información que comparten, puesto que posiblemente una buena cantidad de ellos sencillamente escogen la opción recomendada por su RSO preferida.

\section{Agradecimientos.}

Se agradece a Frederic Gil, Carla Casado, Anthony Francome y Vanessa Renau su ayuda en la recogida de datos.

\section{Fuentes de Financiación.}

Este estudio ha sido parcialmente financiado por una ayuda I+D+I del Ministerio de Economía y Competitividad (referencia: FEM 2012-33505).

\section{Referencias bibliográficas.}

Acquisti, A. \& Gross, R. (2006). Imagined communities: Awareness, information sharing, and privacy on the Facebook. En P. Golle \& G. Danezis (Eds.), Proceedings of 6th Workshop on Privacy Enhancing Technologies (pp. 36-58). Cambridge, UK: Robinson College.

Almansa, A., Fonseca, O. \& Castillo, A. (2013). Redes sociales y jóvenes. Uso de Facebook en la juventud colombiana y española. Revista Comunicar, 20, 127-135. doi: http://dx.doi.org/10.3916/C40-2013-03-03

Bennett, S., Maton, K. \& Kervin, L. (2008). The «digital natives» debate: A critical review of the evidence. British Journal of Educational Technology, 39, 775-786. doi:10.1111/j.1467-8535.2007.00793.x

B³achnio, A., Przepiórka, A. \& Rudnicka, P. (2013). Psychological determinants of using Facebook: A research review. International Journal of Human-Computer Interaction, 29, 775-787. doi: 10.1080/10447318.2013.780868

Boyd, D. (2014). It's complicated: The Social Lives of Networked Teens. Yale University Press.

Boyd, D. M. \& Ellison, N. B. (2007). Social Network Sites: Definition, History, and Scholarship. Journal of Computer-Mediated Communication, 13, 210-230. doi:10.1111/ j.1083-6101.2007.00393.x

Caers, R., De Feyter, T., De Couck, M., Stough, T., Vigna, C. \& Du Bois, C. (2013). Facebook: A literature review. New Media \& 
Society, 15, 982-1002. doi:10.1177/ 1461444813488061.

Casado, C., Oberst, U. \& Carbonell, X. (2015). Facebook: Personalidad y privacidad en los perfiles. Anuario de Psicología, 45, 39-54.

Cheung, C. M. K. \& Lee, M. K. O. (2010). A theoretical model of intentional social action in online social networks. Decision Support Systems, 49, 24-30. doi:10.1016/ j.dss.2009.12.006

Christofides, E., Muise, A. \& Desmarais, S. (2009). Information disclosure and control on Facebook: are they two sides of the same coin or two different processes?. Cyberpsychology \& Behavior, 12, 341-345. doi:10.1089/cpb.2008.0226

Christofides, E., Muise, A. \& Desmarais, S. (2011). Hey Mom, What's on Your Facebook? Comparing Facebook Disclosure and Privacy in Adolescents and Adults. Social Psychological and Personality Science, 3, 48-54. doi: 10.1177/1948550611408619

Christofides, E., Muise, A. \& Desmarais, S. (2012). Risky Disclosures on Facebook: The Effect of Having a Bad Experience on Online Behavior. Journal of Adolescent Research, 27, 714-731. doi: 10.1177/0743558411432635

Davenport, S. W., Bergman, S. M., Bergman, J. Z. \& Fearrington, M. E. (2014). Twitter versus Facebook: Exploring the role of narcissism in the motives and usage of different social media platforms. Computers in Human Behavior, 32, 212-220. doi:10.1016/ j.chb.2013.12.011

Delfour, M., Moreau, A., Laconi, S., Goutaudier, N. \& Chabrol, H. (2014). Utilisation problématique de Facebook à l'adolescence et au jeune âge adulte. Neuropsychiatrie de l'infance et de l'adolescence, 63 (4), 244-249. doi: 10.1016/ j.neurenf.2014.09.005
Ellison, N. B., Steinfield, C. \& Lampe, C. (2007). The Benefits of Facebook «Friends:» Social Capital and College Students' Use of Online Social Network Sites. Journal of Computer-Mediated Communication, 12, 1143-1168. doi: 10.1111/j.10836101.2007.00367.x

García, A., López de Ayala, M. C. \& Catalina, B. (2013). Hábitos de uso en Internet y en las redes sociales de los adolescentes espanyoles. Revista Comunicar, 41, 195-204. doi: 10.3916/C41-2013-19

Instituto Nacional de Tecnologías de la Información (INTECO) (2012). Informe anual. Madrid: Ministerio de Industria, Energía y Turismo. Recuperado de http://goo.gl/5zFozZ Interactive Advertising Bureau (2015). Estudio anual de redes sociales 2015. Recuperado de http://goo.gl/NxMNC2

Junco, R. (2012). The relationship between frequency of Facebook use, participation in Facebook activities, and student engagement. Computers and Education, 58, 162-171. doi:10.1016/j.compedu.2011.08.004

Kalpidou, M., Costin, D. \& Morris, J. (2011). The relationship between Facebook and the well-being of undergraduate college students. CyberPsychology, Behavior \& Social Networking, 14, 183-189. doi: 10.5817/ CP2012-1-2

Kuss, D. \& Griffiths, M. (2011). Online Social Networking and addiction. A review of psychological literature. International Journal of Environmental Research \& Public Health, 8, 3528-3552. doi: 10.3390/ ijerph8093528

Kwon, O. \& Wen, Y. (2010). An empirical study of the factors affecting social network service use. Computers in Human Behavior, 26, 254-263. doi:10.1016/j.chb.2009.04.011

Lewis, K., Kaufman, J. \& Christakis, N. (2008). The Taste for Privacy: An Analysis of 
College Student Privacy Settings in an Online Social Network. Journal of ComputerMediated Communication, 14, 79-100. doi:10.1111/j.1083-6101.2008.01432.x

Linne, J. (2014). Common Uses of Facebook among Adolescents from Different Social Sectors in Buenos Aires City. Comunicar, 22, 189-197. doi:10.3916/C43-2014-19

Merritt, M. (2008). Family Online Safety Guide. Recuperado de http://goo.gl/b98b5Z

Muñoz, R., Ortega, R., Batalla, C., López, M.R., Manresa, J.M. \& Torán, P. (2014). Acceso y uso de nuevas tecnologías entre los jóvenes de educación secundaria, implicaciones en salud. Estudio JOITIC. Atención Primaria, 46, 77-88. doi: 10.1016/ j.aprim.2013.06.001.

Nadkarni, A. \& Hoffmann, S.G. (2012). Why do people use Facebook?. Personality and Individual Differences, 52 (3), 243-249. doi:10.1016/j.paid.2011.11.007

Observatorio Nacional de las Telecomunicaciones y de la Sociedad de la Información (2013). La Sociedad en Red. Informe Anual 2012. Madrid: Ministerio de Energía, Industria y Turismo. Recuperado de http://goo.gl/5LyGfi

Prensky, M. (2001). Digital Natives, Digital Immigrants. On the Horizon, 9 (5), 1-6. doi: http://dx.doi.org/10.1108/10748120110424816

Raacke, J. \& Bonds, J. (2008). MySpace and Facebook: applying the uses and gratifications theory to exploring friendnetworking sites. Cyberpsychology \& Behavior/: The Impact of the Internet, Multimedia and Virtual Reality on Behavior and Society, 11 (2), 169-174. doi:10.1089/ cpb.2007.0056

Renau, V., Carbonell, X. \& Oberst, U. (2012). Redes sociales on-line, género y construcción del self. Aloma, 30 (2), 97-107.
Renau, V., Oberst, U. \& Carbonell, X. (2013). Construcción de la identidad a través de las redes sociales online. Anuario de psicología, 43 (2), 159-170.

Ross, C., Orr, E. S., Sisic, M., Arseneault, J. M., Simmering, M. G. \& Orr, R. R. (2009). Personality and motivations associated with Facebook use. Computers in Human Behavior, 25 (2), 578-586. doi:10.1016/ j.chb.2008.12.024

Selwyn, N. (2009). The digital native - myth and reality. Aslib Proceedings, 61 (4), 364379. doi:10.1108/00012530910973776

Shapiro, L. A. \& Margolin, G. (2014). Growing Up Wired: Social Networking Sites and Adolescent Psychosocial Development. Clinical Child and Family Psychology Review, 17, 1-18. doi: 10.1007/s10567-0130135-1

Shen, J., Brdiczka, O. \& Ruan, Y. (2013). A comparison study of user behavior on Facebook and Gmail. Computers in Human Behavior, 29 (6), 2650-2655. doi:10.1016/ j.chb.2013.06.043

Waters, S. \& Ackerman, J. (2011). Exploring privacy management on Facebook: Motivations and perceived consequences of voluntary disclosure. Journal of ComputerMediated Communication, 17, 101-115. doi:10.1111/j.1083-6101.2011.01559.x

Wilson, R. E., Gosling, S. D. \& Graham, L. T. (2012). A Review of Facebook Research in the Social Sciences. Perspectives on Psychological Science, 7, 203-220. doi:10.1177/1745691612442904

Fecha de recepción: 30-07-2015

Fecha de evaluación: 01-09-2015

Fecha de aceptación: 09-10-2015 